\title{
Gravity and quantum fields in discrete spacetimes
}

\author{
Florian Bauer \\ Deutsches Elektronen-Synchrotron DESY, Hamburg, Germany \\ E-mail: florian.bauer@desy.de
}

Received 27 October 2006

Published 6 June 2007

Online at stacks.iop.org/JPhysA/40/6957

\begin{abstract}
In a $6 \mathrm{D}$ model, where the extra dimensions form a discretized curved disc, we investigate the mass spectra and profiles of gravitons and Dirac fermions. The discretization is performed in detail leading to a star-like geometry. In addition, we use the curvature of the disc to obtain the mass scales of this model in a more flexible way. We also discuss some applications of this setup such as generating small fermion masses.
\end{abstract}

PACS numbers: $\quad 04.50 .+\mathrm{h}, 11.10 . \mathrm{Kk}, 14.60 . \mathrm{Pq}$

\section{Introduction}

We study a six-dimensional (6D) spacetime consisting of a flat four-dimensional (4D) subspace and a disc of constant curvature for the extra dimensions. Furthermore, we discretize the disc in a way that $N$ equidistant lattice sites are situated on the boundary and a single site in the centre of the disc (section 2). Such star-like geometries have been proven useful in various contexts, see for example [1-7]. In this work we investigate the mass spectra and profiles of 4D gravitons and fermions in this setup (sections 3 and 4), which has some nice applications. For instance, we discuss a possibility of hiding extra dimensions similar to [8], and we generate small fermion masses via a discrete version of the wavefunction suppression mechanism [9].

\section{Curved disc geometry}

We consider a 6D model where both extra dimensions form a discretized curved disc (for example, a part of a 2-sphere) while the 4D subspace remains continuous. Before the discretization, the spacetime is described by the line element

$$
\mathrm{d} s^{2}=g_{\mu \nu}\left(x^{M}\right) \mathrm{d} x^{\mu} \mathrm{d} x^{\nu}-\left(1-e r^{2}\right)^{-1} \mathrm{~d} r^{2}-r^{2} \mathrm{~d} \varphi^{2},
$$

where $x^{\mu}$ and $x^{M}$ denote 4D and 6D coordinates, respectively. The position on the disc is fixed by the polar coordinate $\varphi:=x^{6} \in[0,2 \pi]$ and the radial coordinate $r:=x^{5} \in[0, L]$ with $L$ being the coordinate radius of the disc. From (1) we read off the metric components $g_{\mu \nu}\left(x^{M}\right)$ 
of the 4D subspace as well as $g_{55}=-\left(1-e r^{2}\right)^{-1}$ and $g_{66}=-r^{2}$. Note that the parameter $e$ controls the curvature of the disc. For $e>0$ the disc is spherically curved, whereas $e<0$ leads to a hyperbolic disc, and $e=0$ corresponds to a flat disc. Following $[1,10]$ we now decompose the 6D Einstein-Hilbert action,

$$
S=M_{6}^{4} \int \mathrm{d}^{6} x \sqrt{|g|} R=S_{4 \mathrm{D}}+S_{\text {surface }}+S_{\text {mass }}
$$

into three parts, where $R, g$ and $M_{6}$ denote the 6D curvature scalar, the determinant of the metric $g_{M N}$ and the 6D Planck scale, respectively. We find that the $4 \mathrm{D}$ curvature scalar $R_{4 \mathrm{D}}$ in $S_{4 \mathrm{D}}:=M_{6}^{4} \int \mathrm{d}^{6} x \sqrt{|g|}\left(R_{4 \mathrm{D}}+2 e\right)$ contains only the 4D metric $g_{\mu \nu}$ and derivatives with respect to $x^{\mu}$. The surface terms in $S_{4 \mathrm{D}}$ vanish by choosing suitable boundary conditions on the disc, and $S_{\text {mass }}$ is given by

$$
S_{\text {mass }}=M_{6}^{4} \int \mathrm{d}^{6} x \sqrt{|g|} \sum_{c=5,6}\left[-\frac{1}{4} g^{c c} g_{\mu \nu, c}\left(g^{\mu \nu} g^{\alpha \beta}-g^{\mu \alpha} g^{\nu \beta}\right) g_{\alpha \beta, c}\right] .
$$

Let us now introduce 4D graviton fields $h_{\mu \nu}$ on a flat Minkowski metric $\eta_{\mu \nu}=$ diag $(1,-1,-1,-1)$ by the expansion $g_{\mu \nu} \rightarrow \eta_{\mu \nu}+h_{\mu \nu}$. As in [11] we choose a gauge, where we ignore graviphoton and radion excitations, which could result from the $g_{5 M}$ and $g_{6 M}$ components of the metric. Since $\eta_{\mu \nu}$ is constant we have $g_{\mu \nu, A} \rightarrow h_{\mu \nu, A}$ and by expanding $S_{\text {mass }}$ in second order in $h_{\mu \nu}$, we find

$$
\begin{aligned}
S_{\text {mass }} \rightarrow M_{6}^{4} \int & \mathrm{d}^{4} x \mathrm{~d} \varphi \mathrm{d} r\left[+\frac{1}{4} \sqrt{\frac{g_{66}}{g_{55}}} \partial_{r} h_{\mu \nu}\left(\eta^{\mu \nu} \eta^{\alpha \beta}-\eta^{\mu \alpha} \eta^{\nu \beta}\right) \partial_{r} h_{\alpha \beta}\right. \\
+ & \left.\frac{1}{4} \sqrt{\frac{g_{55}}{g_{66}}} \partial_{\varphi} h_{\mu \nu}\left(\eta^{\mu \nu} \eta^{\alpha \beta}-\eta^{\mu \alpha} \eta^{\nu \beta}\right) \partial_{\varphi} h_{\alpha \beta}\right] .
\end{aligned}
$$

Next, we discretize the disc by putting $N$ lattice sites on the boundary and a single site in centre of the disc so that only two points are lying in the radial direction. The coordinate distance between the centre and each point on the boundary is given by the radius $L$ of the disc, which in general differs from its proper radius. On the boundary, the graviton fields are denoted by $h_{\mu \nu}^{i}$ with $i=1, \ldots, N$, and the position is given by $\varphi^{i}=i \cdot \Delta \varphi$, where $\Delta \varphi=2 \pi / N$ is the angular lattice spacing. The graviton field $h_{\mu \nu}^{0}$ in the centre carries the index 0 , and the lattice spacing in the radial direction is just $\Delta r=L$. Formally, we apply the following discretization prescription to $S_{\text {mass }}$ :

$$
\begin{array}{ll}
\partial_{r} h\left(\varphi^{i}\right) \rightarrow \frac{\left(h^{i}-h^{0}\right)}{\Delta r}, & \partial_{\varphi} h\left(\varphi^{i}\right) \rightarrow \frac{\left(h^{i+1}-h^{i}\right)}{\Delta \varphi}, \\
\int \mathrm{d} r f(r) \rightarrow \Delta r \cdot f(L), & \int \mathrm{d} \varphi f(\varphi) \rightarrow \sum_{i=1}^{N} \Delta \varphi \cdot f\left(\varphi^{i}\right),
\end{array}
$$

where the integral $\int \mathrm{d} r$ is replaced by just one summation interval of length $L$ and the summand is evaluated at the position $r=L$, which avoids problems with the derivative $\partial_{\varphi}$ at $r=0$. Thus, we obtain (still non-diagonal) Fierz-Pauli mass terms [12] for the gravitons on the discretized disc that read (with $h_{\mu \nu}^{N+1} \equiv h_{\mu \nu}^{1}$ )

$$
\begin{aligned}
S_{\text {mass }} \rightarrow M_{4}^{2} \int & \mathrm{d}^{4} x \sum_{i=1}^{N}\left[m_{\star}^{2} \cdot\left(h_{\mu \nu}^{i}-h_{\mu \nu}^{0}\right)\left(\eta^{\mu \nu} \eta^{\alpha \beta}-\eta^{\mu \alpha} \eta^{\nu \beta}\right)\left(h_{\alpha \beta}^{i}-h_{\alpha \beta}^{0}\right)\right. \\
& \left.+m^{2} \cdot\left(h_{\mu \nu}^{i+1}-h_{\mu \nu}^{i}\right)\left(\eta^{\mu \nu} \eta^{\alpha \beta}-\eta^{\mu \alpha} \eta^{\nu \beta}\right)\left(h_{\alpha \beta}^{i+1}-h_{\alpha \beta}^{i}\right)\right] .
\end{aligned}
$$


Note that the actual graviton mass scale from the radial derivatives, $m_{\star}$, and respectively from the angular derivatives, $m$, depend on the 4D Planck mass $M_{4}$ of the observer's site (brane):

$$
m_{\star}^{2}:=\frac{M_{6}^{4}}{4 M_{4}^{2}} \cdot \frac{2 \pi \sqrt{1-e L^{2}}}{N}, \quad m^{2}:=\frac{M_{6}^{4}}{4 M_{4}^{2}} \cdot \frac{N}{2 \pi \sqrt{1-e L^{2}}} .
$$

However, the ratio of masses is independent of the Planck scales,

$$
\frac{m_{\star}^{2}}{m^{2}}=\frac{(2 \pi)^{2}}{N^{2}}\left(1-e L^{2}\right),
$$

which also shows that arbitrarily large hierarchies between $m_{\star}$ and $m$ are possible by choosing the disc parameters $e L^{2}$ and $N$ appropriately.

In order to determine the 4D Planck mass $M_{4}$ on the sites we need the proper area of the curved disc, which is given by

$$
A:=\int_{0}^{2 \pi} \mathrm{d} \varphi \int_{0}^{L} \mathrm{~d} r \sqrt{\left|g_{55} g_{66}\right|}=\frac{2 \pi}{e}\left(1-\sqrt{1-e L^{2}}\right) .
$$

We now proceed to discretize $S_{4 \mathrm{D}}$. Since the extra-dimensional disc has a constant curvature it is well motivated that $M_{4}$ should be constant and universal on all sites, too. Thus, the Einstein-Hilbert terms of all $N+1$ sites must have the form

$$
M_{4}^{2} \sum_{i=0}^{N} \int \mathrm{d}^{4} \times R_{4 \mathrm{D}}
$$

which does not depend on $r$ and $\varphi$ anymore. By comparing this term with $S_{4 \mathrm{D}}=M_{6}^{4}$ $\int \mathrm{d}^{6} x \sqrt{|g|} R_{4 \mathrm{D}}$ we find that the 4D Planck scale on the sites is fixed by $M_{4}^{2}=M_{6}^{4} A /(N+1)$, where we used (9) in $S_{4 \mathrm{D}}$ and evaluated the sum in (10). However, we remark that $M_{4}$ is not the (reduced) Planck scale $M_{\mathrm{Pl}}=1 /(8 \pi G) \sim 10^{18} \mathrm{GeV}$ that couples gravity to 4D matter. But $M_{\mathrm{Pl}}$ is determined by integrating out the extra dimensions in the continuum, which means $M_{6}^{4} \int \mathrm{d}^{6} x \sqrt{|g|} R_{4 \mathrm{D}}=M_{\mathrm{Pl}}^{2} \int \mathrm{d}^{4} x R_{4 \mathrm{D}}$ and thus $M_{\mathrm{Pl}}^{2}=M_{6}^{4} A=(N+1) M_{4}^{2}$.

\section{Graviton mass spectrum}

We omit to show the kinetic terms for the 4D gravitons $h_{\mu \nu}^{i}$. They just follow from applying the graviton expansion to the Einstein-Hilbert terms in (10), see e.g. [13]. To determine the graviton mass spectrum, we have to diagonalize $S_{\text {mass }}$ in (6) by a unitary transformation. If we denote the graviton mass eigenstates by $H_{\mu \nu}^{n}$, corresponding to the masses $M_{n}$, we find the following relations for the eigenvectors:

$$
\begin{aligned}
& H_{\mu \nu}^{0}=\frac{1}{\sqrt{N+1}} \sum_{i=0}^{N} h_{\mu \nu}^{i}, \\
& H_{\mu \nu}^{p}=\frac{1}{\sqrt{N}} \sum_{i=1}^{N}\left[\sin \left(2 \pi \frac{p}{N}\right)+\cos \left(2 \pi \frac{p}{N}\right)\right] \cdot h_{\mu \nu}^{i}, \\
& H_{\mu \nu}^{N}=\frac{1}{\sqrt{N(N+1)}}\left[-N \cdot h_{\mu \nu}^{0}+\sum_{i=1}^{N} h_{\mu \nu}^{i}\right],
\end{aligned}
$$

where $p=1, \ldots, N-1$. The eigenvalues $M_{n}$ are respectively given by

$$
M_{0}^{2}=0, \quad M_{p}^{2}=m_{\star}^{2}+4 m^{2} \sin ^{2} \frac{\pi p}{N}, \quad M_{N}^{2}=(N+1) m_{\star}^{2} .
$$


From these results we observe that the zero-mode $H_{\mu \nu}^{0}$ has a flat profile and is equally located on all sites, whereas the mode $H_{\mu \nu}^{N}$ with squared mass $(N+1) m_{\star}^{2}$ is peaked on the centre site with equal support on the boundary sites. The modes $H_{\mu \nu}^{p}$ with $p=1, \ldots, N-1$ are located only on the boundary with a typical finite Kaluza-Klein (KK) mass spectrum that has been shifted by $m_{\star}^{2}$. In the limit $m \ll m_{\star}$ the masses of the states $H_{\mu \nu}^{p}$ in (12) become degenerate, and for $N \gg 1$ the mode $H_{\mu \nu}^{N}$ becomes very heavy. Note that the latter case can be realised by a sufficiently large negative curvature of the disc, which is a clear advantage over a flat disc model.

Finally, we mention that a scenario related to ours has been discussed recently in the context of multi-throat geometries [8]. It was shown that large extra dimensions can be hidden in the sense that the occurrence of massive KK modes is shifted to energies much higher than the compactification scale of the extra dimension, which helps evading limits on KK particles. In our model this behaviour can be observed for the modes $H_{\mu \nu}^{n>0}$ in the limit $m_{\star} \gg m$, too.

\section{Fermions on the disc}

Let us now investigate the incorporation of Dirac fermions into the discretized disc model of section 2. As for the graviton case, we start with a 6D Dirac fermion $\Psi$ in the continuum. Using the vielbein formalism, the corresponding action $S$ on the curved disc reads

$$
S=\int \mathrm{d}^{6} x \sqrt{|g|}\left[\frac{1}{2} \mathrm{i}\left(\bar{\Psi} G^{A} V_{A}^{M} \nabla_{M} \Psi-\overline{\nabla_{M} \Psi} V_{A}^{M} G^{A} \Psi\right)\right],
$$

where we denote $6 \mathrm{D}$ Lorentz indices by $A, B, \ldots$ and general coordinate indices by $M, N, \ldots$. Moreover, $G^{A}$ are $6 \mathrm{D}$ Dirac matrices, and for the barred spinor $\bar{\Psi}$ we use the abbreviation $\bar{\Psi}=\Psi^{\dagger} G^{0}$. The vielbein components $V_{A}^{M}\left(x^{N}\right)$ follow from the relation $g^{M N}=V_{A}^{M} V_{B}^{N} \eta^{A B}$, which connects the Lorentz coordinate system with the general coordinate system. For the diagonal metric (1), we find $V_{A}^{M}=\delta_{A}^{M}$ with the exceptions $V_{A=5}^{M=5}=\sqrt{\left|g^{55}\right|}=: V_{5}$ and $V_{A=6}^{M=6}=\sqrt{\left|g^{66}\right|}=: V_{6}$. On a curved spacetime, the covariant derivative $\nabla_{M}=\partial_{M}+\Gamma_{M}$ for spinors contains in addition to the usual partial derivative $\partial_{M}$ also the spin connection $\Gamma_{M}=\frac{1}{8}\left[G^{A}, G^{B}\right] V_{A}^{N} V_{B N ; M}$.

To determine the form of the 6D $\gamma$-matrices [14] let us first look at the 4D case, where the $\gamma$-matrices are given by

$$
\gamma^{0}=\left[\begin{array}{cc}
0 & 1_{2} \\
1_{2} & 0
\end{array}\right], \quad \gamma^{k}=\left[\begin{array}{cc}
0 & \sigma^{k} \\
-\sigma^{k} & 0
\end{array}\right], \quad \gamma^{5}=\mathrm{i} \gamma^{0} \gamma^{1} \gamma^{2} \gamma^{3} .
$$

Here, $k=1, \ldots, 3$ and $\sigma^{k}$ denote the Pauli matrices. In five dimensions, the number of spinor components is still four and the corresponding $\gamma$-matrices are simply given by $\Gamma^{0}=\gamma^{0}, \Gamma^{k}=\gamma^{k}$ and $\Gamma^{5}=\mathrm{i} \gamma^{5}=-\left(\Gamma^{5}\right)^{\dagger}$. In six dimensions, however, the Dirac algebra is eight dimensional, where we use the following set of $\gamma$-matrices:

$$
\begin{aligned}
G^{0} & =\left[\begin{array}{cc}
0 & 1_{4} \\
14 & 0
\end{array}\right], \quad G^{6}=\left[\begin{array}{cc}
0 & \Gamma^{0} \\
-\Gamma^{0} & 0
\end{array}\right]=-\left(G^{6}\right)^{\dagger}, \\
G^{n} & =\left[\begin{array}{cc}
0 & \Gamma^{0} \Gamma^{n} \\
-\Gamma^{0} \Gamma^{n} & 0
\end{array}\right]=-\left(G^{n}\right)^{\dagger}, \quad n=1,2,3,5,
\end{aligned}
$$

which fulfil the Cifford algebra $\left\{G^{A}, G^{B}\right\}=2 \eta^{A B} \cdot 1_{8}$.

From the form of the vielbeins and the $\gamma$-matrices, it follows that all spin connection components $\Gamma_{M}$ vanish except for $\Gamma_{6}=\frac{1}{4}\left[G^{5}, G^{6}\right] \sqrt{1-e r^{2}}$. Because of $\Gamma_{6}=-\Gamma_{6}^{\dagger}$, the terms involving $\Gamma_{6}$ in (15) cancel. 
Let us now diagonalize the fermion action by the substitution $\Psi=G^{6} \Phi$. Then the decomposition of the eight-component spinor $\Phi=\left(\Phi_{a}, \Phi_{b}\right)^{\mathrm{T}}$ into two four-component spinors $\Phi_{a}, \Phi_{b}$ yields in (15)

$$
\begin{aligned}
\mathrm{i} \bar{\Psi} G^{A} V_{A}^{M} \nabla_{M} \Psi & =\mathrm{i}\left[\overline{\Phi_{a}}, \overline{\Phi_{b}}\right] \times\left[\left(\begin{array}{cc}
\gamma^{0} & 0 \\
0 & \gamma^{0}
\end{array}\right) \partial_{0}+\left(\begin{array}{cc}
-\gamma^{k} & 0 \\
0 & \gamma^{k}
\end{array}\right) \partial_{k}\right. \\
& \left.+\left(\begin{array}{cc}
-\mathrm{i} \gamma^{5} & 0 \\
0 & +\mathrm{i} \gamma^{5}
\end{array}\right) V_{5} \partial_{5}+\left(\begin{array}{cc}
1 & 0 \\
0 & -1
\end{array}\right) V_{6} \partial_{6}\right] \times\left[\begin{array}{c}
\Phi_{a} \\
\Phi_{b}
\end{array}\right]
\end{aligned}
$$

with $\overline{\Phi_{a, b}}=\Phi_{a, b}^{\dagger} \gamma^{0}$. From the last line one can read off that $\Phi_{a}$ corresponds to $\Phi_{b}$ but with negative energy; therefore we will work only with $\Phi_{b}$ in the following. If we now denote the left- and right-handed components of $\Phi_{b}$ by $\Phi_{\mathrm{L}, \mathrm{R}}:=\frac{1}{2}\left(1 \mp \gamma^{5}\right) \Phi_{b}$, then the full action for $\Phi_{b}$ can be written in the form

$$
\begin{aligned}
S=\int \mathrm{d}^{6} x \sqrt{|g|}[ & \frac{1}{2} \mathrm{i}\left(\overline{\Phi_{b}} \gamma^{\mu} \partial_{\mu} \Phi_{b}-\overline{\partial_{\mu} \Phi_{b}} \gamma^{\mu} \Phi_{b}\right) \\
& \left.-V_{5} \frac{1}{2}\left(\overline{\Phi_{\mathrm{L}}} \partial_{5} \Phi_{\mathrm{R}}+\overline{\partial_{5} \Phi_{\mathrm{R}}} \Phi_{\mathrm{L}}\right)-\mathrm{i} V_{6} \frac{1}{2}\left(\overline{\Phi_{\mathrm{L}}} \partial_{6} \Phi_{\mathrm{R}}-\overline{\partial_{6} \Phi_{\mathrm{R}}} \Phi_{\mathrm{L}}\right)\right],
\end{aligned}
$$

where we have applied the boundary conditions of section 2 after integration by parts.

Since $6 \mathrm{D}$ spinors have mass dimension $\frac{5}{2}$, we have to rescale them in order to obtain usual 4D spinors. As in the graviton case we integrate the kinetic terms over the extra dimensions and apply a similar discretization procedure as in section 2 , which means

$$
\int \mathrm{d}^{6} x \sqrt{|g|} \frac{1}{2} \mathrm{i} \overline{\Phi_{b}} \gamma^{\mu} \partial_{\mu} \Phi_{b} \rightarrow \sum_{j=0}^{N} \frac{A}{N+1} \int \mathrm{d}^{4} x \frac{1}{2} \overline{\mathrm{i}} \overline{\Phi_{b}^{j}} \gamma^{\mu} \partial_{\mu} \Phi_{b}^{j},
$$

where $A$ is the proper area given in (9). Finally, we absorb the factor $A /(N+1)$ into the fermion fields $\chi:=\Phi_{b} \sqrt{A /(N+1)}$ and subsequently apply the discretization prescriptions (5) with $h_{\mu \nu}$ replaced by $\chi$. As a result, we obtain the action for $N+14 \mathrm{D}$ fermions $\left(\chi^{N+1} \equiv \chi^{1}\right)$

$$
\begin{aligned}
S=\sum_{j=0}^{N} \int \mathrm{d}^{4} x & \frac{1}{2} \mathrm{i}\left(\overline{\chi^{j}} \gamma^{\mu} \partial_{\mu} \chi^{j}-\overline{\partial_{\mu} \chi^{j}} \gamma^{\mu} \chi^{j}\right) \\
& \left.-\sum_{j=1}^{N} \int \mathrm{d}^{4} x \cdot m_{\star}\left(\overline{\chi_{\mathrm{L}}^{j}}\left(\chi_{\mathrm{R}}^{j}-\chi_{\mathrm{R}}^{0}\right)+\overline{\left(\chi_{\mathrm{R}}^{j}\right.}-\overline{\chi_{\mathrm{R}}^{0}}\right) \chi_{\mathrm{L}}^{j}\right) \\
& -\sum_{j=1}^{N} \int \mathrm{d}^{4} x \cdot \mathrm{i} m\left(\overline{\chi_{\mathrm{L}}^{j}}\left(\chi_{\mathrm{R}}^{j+1}-\chi_{\mathrm{R}}^{j}\right)-\left(\overline{\chi_{\mathrm{R}}^{j+1}}-\overline{\chi_{\mathrm{R}}^{j}}\right) \chi_{\mathrm{L}}^{j}\right)
\end{aligned}
$$

with the mass scales $m_{\star}:=2 \pi L(N+1) /(A N)$ and $m:=L(N+1) /\left(A \sqrt{1-e L^{2}}\right)$. Hence, the ratio $m_{\star}^{2} / m^{2}$ is the same ratio as in (8) for the gravitons. Next, we apply a bi-unitary transformation relating the states $\chi$ to the mass eigenstates $\psi$ :

$$
\begin{aligned}
& \overline{\chi_{\mathrm{L}}^{0}}=\overline{\psi_{\mathrm{L}}^{0}}, \quad \overline{\chi_{\mathrm{L}}^{j}}=\frac{1}{\sqrt{N}} \sum_{n=1}^{N} \exp \left(+2 \pi \mathrm{i} \cdot j \frac{n}{N}\right) \overline{\psi_{\mathrm{L}}^{n}}, \\
& \chi_{\mathrm{R}}^{0}=\frac{1}{\sqrt{N+1}} \psi_{\mathrm{R}}^{0}-\frac{N}{\sqrt{N(N+1)}} \psi_{\mathrm{R}}^{N}, \\
& \chi_{\mathrm{R}}^{j}=\frac{1}{\sqrt{N}} \sum_{n=1}^{N-1} \exp \left(-2 \pi \mathrm{i} \cdot j \frac{n}{N}\right) \psi_{\mathrm{R}}^{n}+\frac{1}{\sqrt{N+1}} \psi_{\mathrm{R}}^{0}+\frac{1}{\sqrt{N(N+1)}} \psi_{\mathrm{R}}^{N} .
\end{aligned}
$$


The corresponding mass spectrum contains one massless fermion $\psi^{0}$, one heavy fermion $\psi^{N}$ with mass $m_{\star} \sqrt{N+1}$ and $N-1$ fermions $\psi^{1}, \ldots, \psi^{N-1}$ with squared absolute mass values $m_{\star}^{2}+4 m^{2} \sin ^{2}\left(\frac{\pi n}{N}\right)+2 m_{\star} m \sin \left(\frac{2 \pi n}{N}\right)$. In contrast to the graviton mass spectrum (14), here we find an additional interference term $\propto m_{\star} m$, which can be removed by a slightly modified discretization procedure for the angular direction. Instead of $\partial_{6} \chi \rightarrow\left(\chi^{j+1}-\chi^{j}\right) / \Delta \varphi$, we use the prescription $\partial_{6} \chi \rightarrow \mathrm{i}\left(\chi^{j+\frac{1}{2}}-\chi^{j-\frac{1}{2}}\right) / \Delta \varphi$. This does not change the zero mode or the heavy mode, but the transformations in (23) now lead to the mass spectrum $m_{\star}^{2}+4 m^{2} \sin ^{2}\left(\frac{\pi n}{N}\right)$ for the modes $\psi^{1}, \ldots, \psi^{N-1}$, which has exactly the same structure as that of the gravitons in (14).

Our results for the fermions on the discretized disc can be applied directly to generate small fermion masses. For this purpose we put the standard model of particles (SM) on the centre site of our disc. In this place, the left-handed SM lepton doublet $\ell$ may couple to the $4 \mathrm{D}$ component $\chi_{\mathrm{R}}^{0}$ of the 6D Dirac field and to the vacuum expectation value $\langle H\rangle$ of the Higgs doublet via an Yukawa interaction term schematically given by $\bar{\ell}\langle H\rangle \chi_{\mathrm{R}}^{0}$. Now, a large number $N \sim 10^{24}$ of lattice sites lets $\Phi^{N}$ decouple due to its large mass $m_{\star} \sqrt{N+1}$, and (23) shows that the right-handed fermion $\chi_{\mathrm{R}}^{0}$ on the centre site essentially consists only of the zero-mode $\psi_{\mathrm{R}}^{0}$ with a tiny weight factor $1 / \sqrt{N+1}$. Thus, the Yukawa interaction of $\ell$ with $\chi_{\mathrm{R}}^{0}$,

$$
\bar{\ell}\langle H\rangle \chi_{\mathrm{R}}^{0} \sim \frac{1}{\sqrt{N+1}} v_{\mathrm{L}}\langle H\rangle \psi_{\mathrm{R}}^{0},
$$

leads to a strong suppression of the SM neutrino $\left(v_{\mathrm{L}}\right)$ mass, representing a discrete version [1] of the wavefunction suppression mechanism in continuous higher dimensions [9].

\section{Conclusions}

Our 6D model with a discretized extra-dimensional curved disc leads to mass spectra that have the same structure for gravitons and fermions. Moreover, the special discretization of the disc allows that the ratio of mass scales in the spectra can be adjusted in a flexible manner by the parameters of the disc. It is thus possible to obtain a gap between the zero mode and the first massive mode that is much larger than the gap between the other massive modes. We have also discussed the generation of small SM fermion masses in this setup. Finally, we mention that the strong coupling regime of this model and a more refined scenario including warping were investigated in [1], where some of our results have been applied, too.

\section{References}

[1] Bauer F, Hällgren T and Seidl G 2006 Discretized gravity in 6D warped space Preprint hep-th/0608176

[2] Hällgren T and Ohlsson T 2006 J. Cosmol. Astropart. Phys. (Preprint hep-ph/0510174)

Hällgren T 2006 Kaluza-Klein dark matter from deconstructed universal extra dimensions Preprint hep-ph/0610367

[3] Seidl G 2006 Discretized gravity on the hyperbolic disk Preprint hep-th/0610291

[4] Bauer F, Lindner M and Seidl G 2004 J. High Energy Phys. JHEP05(2004)026 (Preprint hep-th/0309200)

[5] Witten E 2002 Deconstruction, $G_{2}$ holonomy, and doublet-triplet splitting Preprint hep-ph/0201018

[6] Fulling S A 2005 Local spectral density and vacuum energy near a quantum graph vertex Preprint math.SP/0508335

[7] Bellazzini B and Mintchev A 2006 J. Phys. A: Math. Gen. 3911101 (Preprint hep-th/0605036)

[8] Kim H D 2006 J. High Energy Phys. JHEP01(2006)090 (Preprint hep-th/0510229)

[9] Dienes K R, Dudas E and Gherghetta T 1999 Nucl. Phys. B 55725 (Preprint hep-ph/9811428)

Arkani-Hamed N, Dimopoulos S, Dvali G R and March-Russel J 2002 Phys. Rev. D 65024032 (Preprint hep-ph/9811448)

[10] Bauer F 2006 The cosmological constant and discrete space-times Preprint hep-th/0610178 
[11] Arkani-Hamed N and Schwartz M D 2004 Phys. Rev. D 69104001 (Preprint hep-th/0302110)

Randall L, Schwartz M D and Thambyapillai S 2005 J. High Energy Phys. JHEP10(2005)110 (Preprint hep-th/0507102)

[12] Fierz M and Pauli W 1939 Proc. R. Soc. A 173211

[13] 't Hooft G and Veltman M J G 1974 Ann. Poincaré Phys. Theor. A 2069

[14] Pilaftsis A 1999 Phys. Rev. D 60105023 (Preprint hep-ph/9906265) 